\title{
Laser-Induced Ejection of Millimeter-Sized Liquid Droplet from Metal Surface with a 1 Joule/Pulse ns-Laser
}

\author{
Hiroyuki Niino ${ }^{1}$, Yoshinori Kato², Takashi Kurita², and Toshiyuki Kawashima² \\ ${ }^{1}$ National Institute of Advanced Industrial Science and Technology (AIST), Tsukuba, Ibaraki, $305-$ \\ 8565 Japan \\ E-mail: E-mail: niino.hiro@aist.go.jp \\ ${ }^{2}$ Industrial Development Center, Hamamatsu Photonics K. K., Hamamatsu, Shizuoka, \\ 431-1202 Japan
}

\begin{abstract}
The laser-induced cleaning of a liquid droplet from the surface of a solid substrate is an attractive application of laser technology. We report the laser-induced ejection of an organic carbonate liquid droplet from an aluminum surface using a one Joule per pulse class nanosecond-pulsed laser. The dynamic behavior of the liquid desorption was observed with a high-speed CCD camera. The use of high-power laser equipment makes it possible to perform single-pass cleaning of large areas on a solid surface with a non-contact process.

DOI: 10.2961/jlmn.2018.03.0007
\end{abstract}

Keywords: laser cleaning, aluminum metal plate, organic carbonate liquid, high-speed CCD camera observation

\section{Introduction}

Laser-induced explosive vaporization is an attractive surface cleaning technique for removing liquid contaminants from solid substrates [1]. Explosive vaporization of a liquid at the surface of a metal film by pulsed laser irradiation was reported by A. C. Tam [2, 3]. Their pioneering work used an optical probing technique to investigate liquid water and alcohols, elucidating the dynamics of transient liquid behavior at the metal-liquid interface. This provides a useful surface cleaning process for practical use. Theoretical analysis of explosive vaporization was performed by B. J. Garrison [4]. Thermal expansion due to laser heating was simulated by molecular dynamics modeling.

This technique can be applied, for instance, to cell assembling process of lithium-ion secondary batteries [5]. Electrolyte droplets are often deposited around an injection hole during electrolyte filling into an aluminum case, which induces porosity defects when a sealing cap is laserwelded to the aluminum case; these droplets should be removed before the laser-welding process.

In the present work, the laser-induced ejection of a millimeter-sized liquid droplet from a metal surface using a 1 Joule per pulse class nanosecond-pulsed laser was observed by monitoring the transient dynamics with a high-speed CCD camera. An organic carbonate liquid medium was chosen because the industrial manufacturing of lithium-ion batteries requires an effective non-contact cleaning method. As discussed in the following sections, it is possible to perform non-contact single-pass cleaning of large areas on a solid surface using high-power laser equipment.

\section{Experimental Method}

We used an aluminum plate (A1050, purity: $>99.5 \%, 1$ mm thick) as the solid substrate. The surface of the plate originally had hairline polishing finish. The sample plate was used as received, without further cleaning treatment before the laser experiment. An organic carbonate (mixture of equal amounts of ethyl-methyl-carbonate and ethylenecarbonate) liquid contaminant was selected. ca. $10 \times 5 \mathrm{~mm}$ liquid droplets were prepared on the metal surface using a $10 \mu \mathrm{L}$ dropper.

The beam of a prototype nanosecond-pulsed laser (Hamamatsu Photonics K. K., wavelength: 1053 nm, pulseduration: $5.7 \mathrm{~ns}$, pulse repetition rate: $1 \mathrm{~Hz}$, laser pulse energy: $3 \mathrm{~J}$ pulse $^{-1}-0.5 \mathrm{~J}$ pulse $^{-1}$ ) was incident on the surface at an angle of $45^{\circ}$ in ambient air (Fig. 1). As the laser beam (original squire-shape beam size: $16 \times 16 \mathrm{~mm}$ ) was not focused with a lens onto the target plate, the laser fluence on the surface was varied by changing the laser pulse energy of the laser equipment.

The laser-induced ejection of the liquid droplet was observed using a high-speed CCD camera (Casio Computer Co., Ltd., model: EXILIM EX-F1, frame rates: 30 fps and $300 \mathrm{fps}$ ). The surface morphological damage was observed using SEM (Hitachi High-Technologies Co., model: TM3030, attachment: Energy Dispersive X-ray Spectrometer (Brucker: Quantax70)) and a 3D morphological measurement system (Keyence Co., model: VR-3000). The transmission absorption spectrum of the liquid in the UVvisible-NIR region was obtained using a scanning spectrophotometer (Shimadzu Co., model: UV-3100PC) 


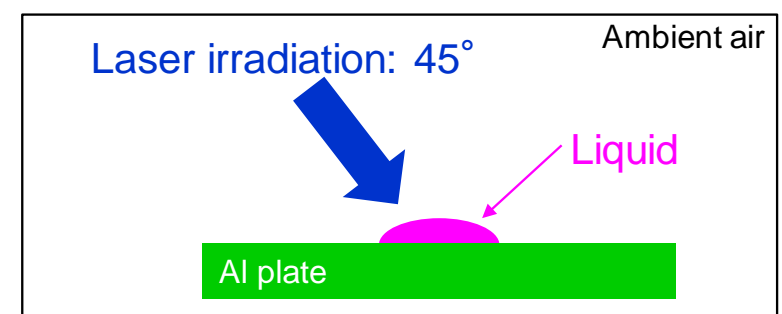

Fig. 1 Side-view illustration of the experimental setup of laser irradiation for liquid droplet ejection

(original laser beam size: 16 x $16 \mathrm{~mm}$ ).

\section{Results and Discussion}

\subsection{Laser-induced ejection of millimeter-sized liquid} droplets from an aluminum surface

Figure 2 shows a top-view CCD camera image of the aluminum surface before and after laser irradiation at a fluence of $0.8 \mathrm{~J} \mathrm{~cm}^{-2}$ (CCD frame rate: $30 \mathrm{fps}$ ). First, a 10 $\mu \mathrm{L}$ liquid droplet was placed on the aluminum plate (Fig. 1(a)). After the single-shot irradiation (Fig. 1(b)), most of the liquid droplet was desorbed from the surface. Several smaller droplets of the liquid were re-deposited around the irradiated area (orange arrows in Fig. 1(b)). After a subsequent five-shot irradiation, the original droplet was eliminated from the surface (Fig. 1(c)). Thus, single-pass cleaning of a $1 \mathrm{~cm}^{2}$ area on the solid surface was performed by laser irradiation.
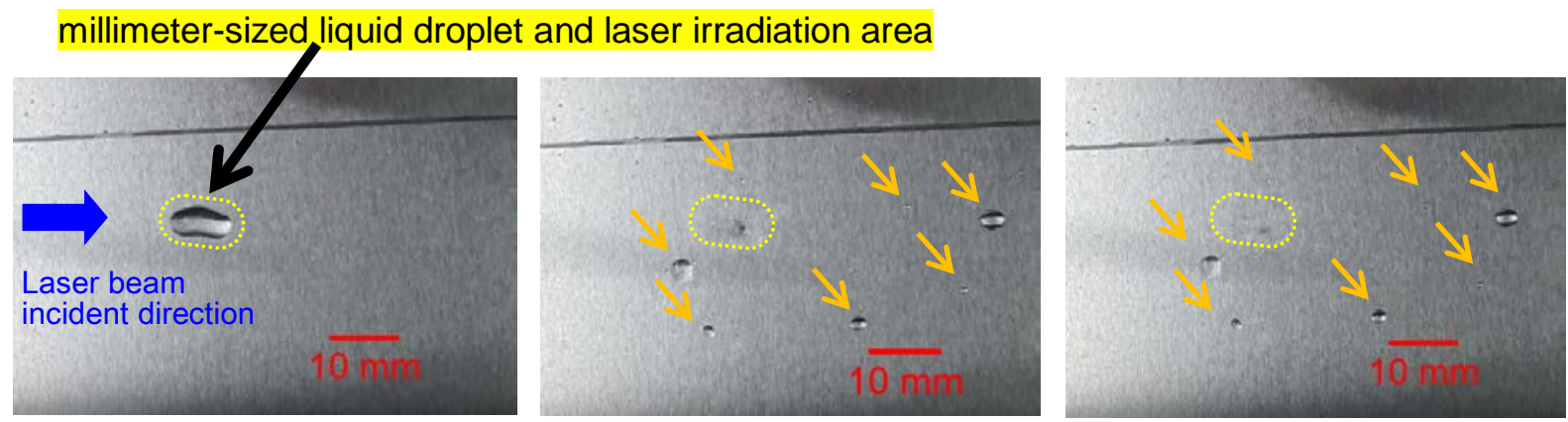

Fig. 2 Laser-induced ejection of a millimeter-sized liquid droplet from an aluminum metal surface with a nanosecond-laser at a fluence of $0.8 \mathrm{~J} \mathrm{~cm}^{-2}$ (original laser beam size: $16 \times 16 \mathrm{~mm}$ ). The CCD frame rate was $30 \mathrm{fps}$. (a) Before the laser irradiation (arrow: original liquid droplet (volume: $10 \mu \mathrm{L}$ ), blue arrow: laser beam incident direction). (b) After 1-pulse laser irradiation (orange arrows indicate desorbed and redeposited smaller droplets on the surface). (c) After 5-pulse laser irradiation (orange arrows again indicate desorbed and redeposited smaller droplets on the surface).

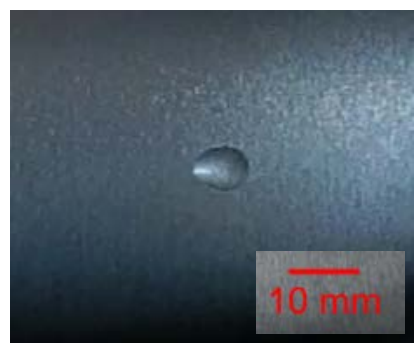

(a)

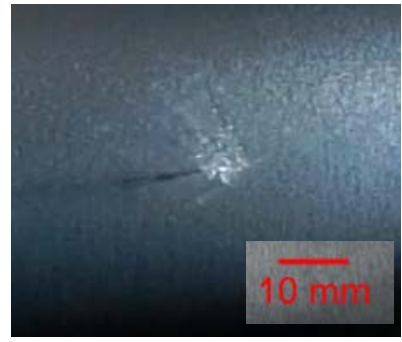

(e)

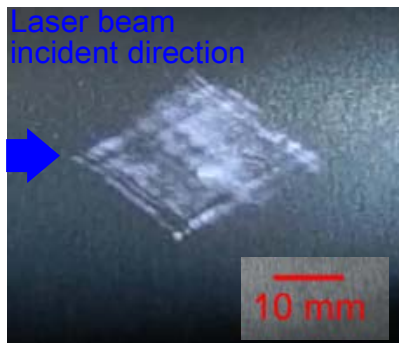

(b)

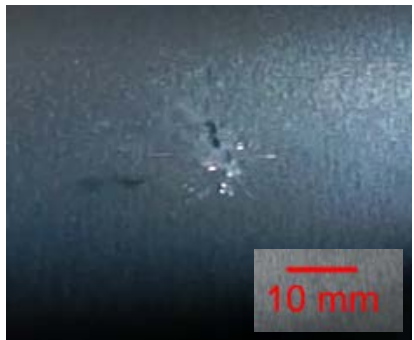

(f)

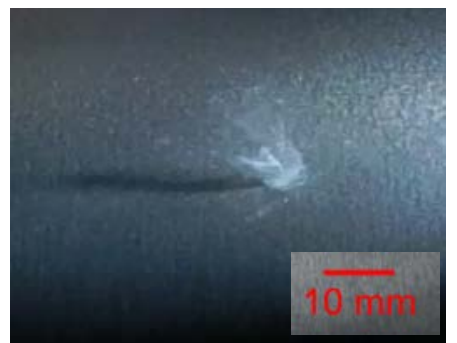

(c)

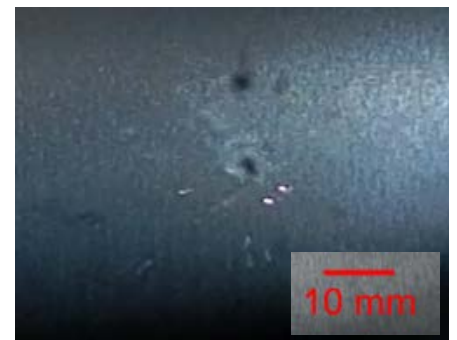

(g)

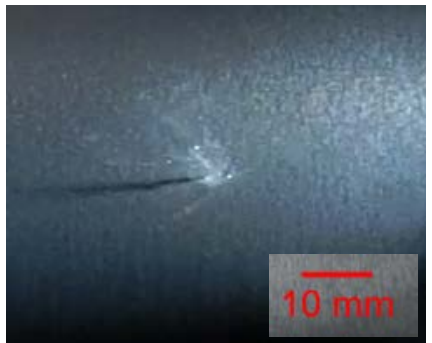

(d)

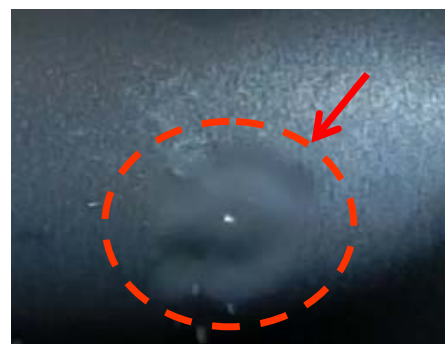

(h)

Fig. 3 Laser-induced ejection of a millimeter-sized liquid droplet from an aluminum metal surface with a nanosecond-laser at a fluence of $0.8 \mathrm{~J} \mathrm{~cm}^{-2}$ (original laser beam size: $16 \times 16 \mathrm{~mm}$ ). The CCD frame rate was $300 \mathrm{fps}$. (a) Before the laser irradiation. Original liquid droplet volume: $10 \mu \mathrm{L}$. (b) During the laser irradiation of the first pulse. The bright square region is the laser-irradiated area. (blue arrow: laser beam incident direction) (c) $3.3 \mathrm{~ms}$ after the laser irradiation (the next frame image after (b)). (d) 6.7 ms after the laser irradiation. (e) $10 \mathrm{~ms}$ after the laser irradiation. (f) $16.7 \mathrm{~ms}$ after the laser irradiation. (g) $33.3 \mathrm{~ms}$ after the laser irradiation. (h) $53.3 \mathrm{~ms}$ after the laser irradiation (red arrow and circle: a liquid droplet desorbed from the plate and re-deposited onto the CCD camera lens surface). 
Figure 3 shows top-view pictures of laser-induced ejection of a millimeter-sized liquid droplet observed with the CCD camera (frame rate: $300 \mathrm{fps}$ ). The camera was placed perpendicular to the irradiation area at a distance of $20 \mathrm{~cm}$ from the target surface. After the laser irradiation of Fig. 3(b), splashes of tiny liquid droplets from the surface are clearly observable in Fig. 3(c). Liquid jets from the surface were detected up to $10 \mathrm{~ms}$ after the laser irradiation. In Figs. 3(f)-3(h), small droplets of ejected liquid are observable. Notably, a droplet desorbed from the surface was deposited on the surface of the CCD camera lens, indicating that the droplet ejection speed in air was approximately $4 \mathrm{~m} \mathrm{~s}^{-1}$.

\subsection{Mechanism of laser ejection behavior}

By laser irradiation of the bare aluminum plate without a liquid droplet in ambient air, the surface melting threshold fluence of the plate was estimated to be $1 \mathrm{~J} \mathrm{~cm}^{-2}$ (Table 1). After laser irradiation with a fluence below $1 \mathrm{~J} \mathrm{~cm}^{-2}$ in air, no morphological changes were observed on the surface of the aluminum plate.

On the other hand, the threshold fluence for the ejection of the liquid from the aluminum surface was observed to be $0.6 \mathrm{~J} \mathrm{~cm}^{-2}$. Therefore, laser fluences of 0.6 and $0.8 \mathrm{~J} \mathrm{~cm}^{-2}$ (at and above the liquid ejection threshold, but below the melting threshold for the metal) were employed in this experiment.

Table 1 Threshold fluences of laser irradiation phenomena.

\begin{tabular}{cc}
\hline $\begin{array}{c}\text { Laser irradiation phenome- } \\
\text { non in air }\end{array}$ & Threshold laser fluence \\
\hline $\begin{array}{c}\text { Melting of bare aluminum } \\
\text { plate without liquid }\end{array}$ & $1 \mathrm{~J} \mathrm{~cm}^{-2}$ \\
$\begin{array}{c}\text { Ejection of carbonate liquid } \\
\text { from aluminum plate }\end{array}$ & $0.6 \mathrm{~J} \mathrm{~cm}^{-2}$ \\
\hline
\end{tabular}

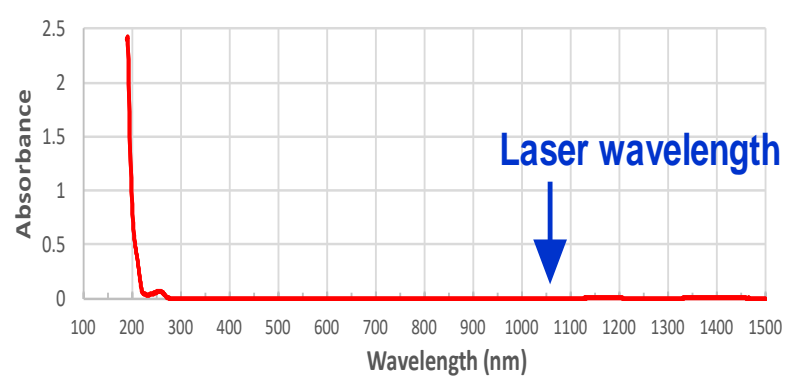

Fig. 5 Transmission absorption spectrum of the liquid in the UVvisible-near IR region (liquid thickness: $0.1 \mathrm{~mm}$ ).

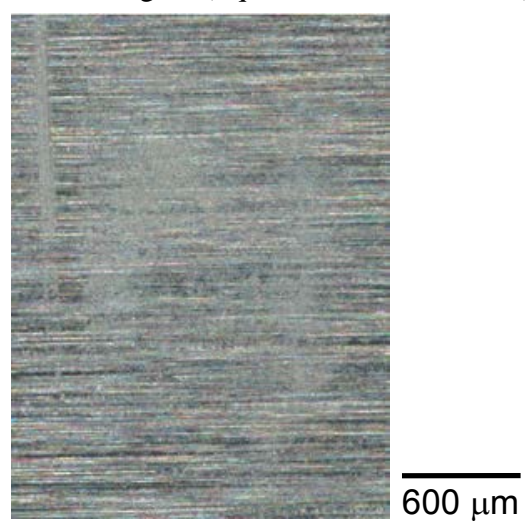

(a)

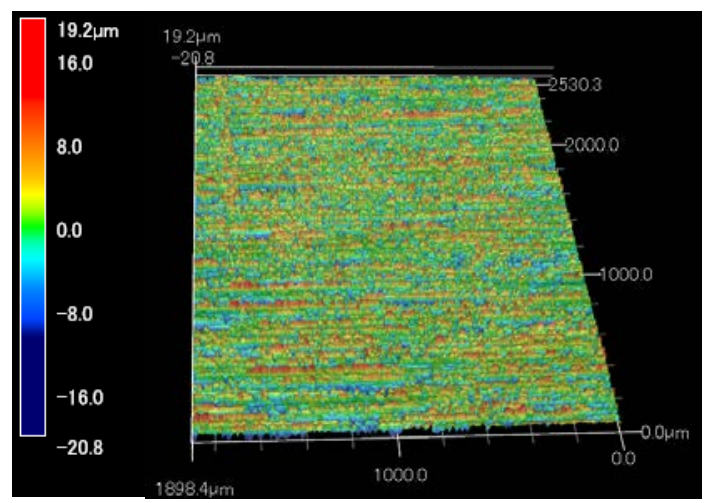

(b)

Fig. 6 Morphological damage observed in the droplet-ejected area of the metal surface after liquid ejection by single-pulse laser irradiation at a fluence of $0.8 \mathrm{~J} \mathrm{~cm}^{-2}$. (a) Top-view of optical micrograph, (b) 3D morphological measurement.

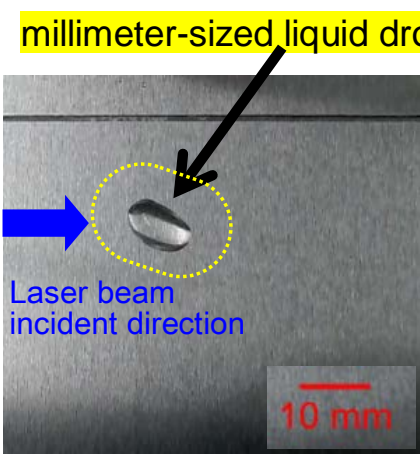

(a)

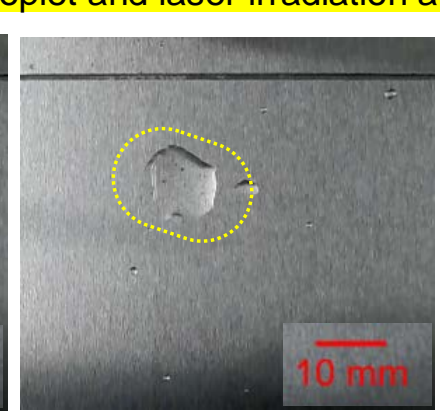

(b)

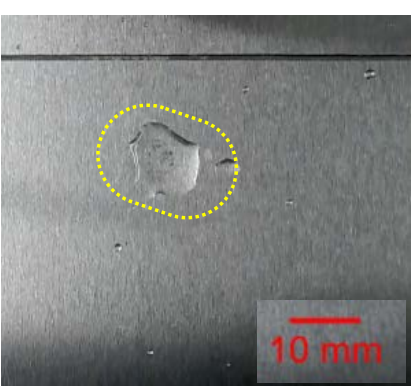

(c)

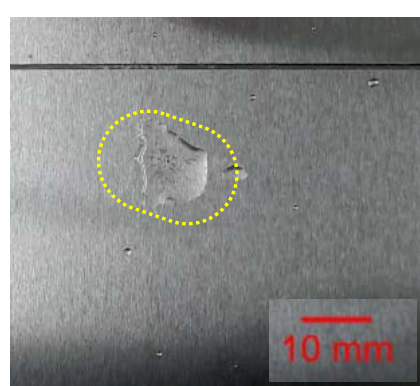

(d)

Fig. 4 Laser-induced ejection of a millimeter-sized liquid droplet from an aluminum metal surface using a ns-laser at a fluence of $0.6 \mathrm{~J}$ $\mathrm{cm}^{-2}$ (original laser beam size: 16 × $16 \mathrm{~mm}$ ). The CCD frame rate was $30 \mathrm{fps}$. (a) Before laser irradiation (arrow: original liquid droplet (volume: $10 \mu \mathrm{L}$ ), blue arrow: laser beam incident direction). (b) After 1-pulse laser irradiation (orange arrows: ejected and redeposited microdroplets on the surface). (c) After 2-pulse laser irradiation (orange arrows: ejected and re-deposited droplets on the surface). (d) After 5-pulse laser irradiation (orange arrows: ejected and re-deposited droplets on the surface). 
(a)

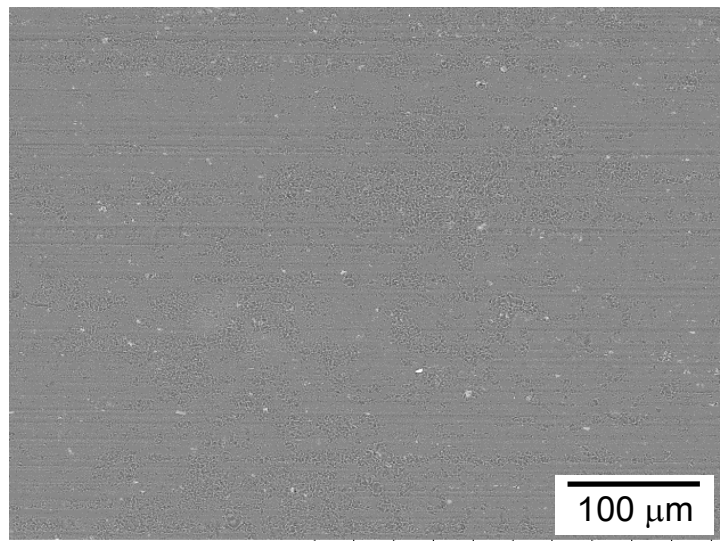

(b)

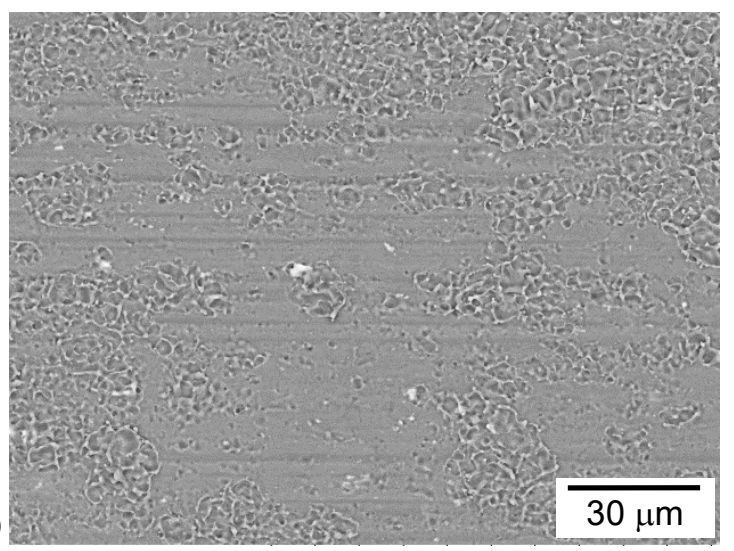

(c)

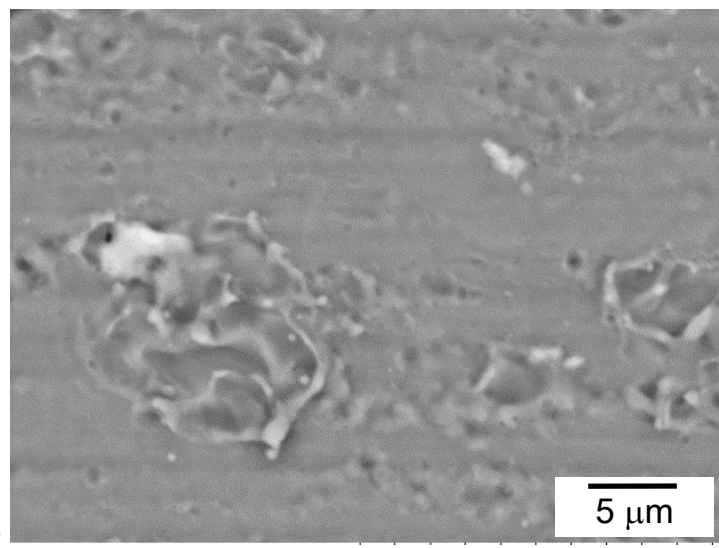

Fig. 7 SEM images of the ejected droplet area on the aluminum surface at three different magnifications (after single-pulse laser irradiation at a fluence of $0.8 \mathrm{~J} \mathrm{~cm}^{-2}$ ).

The liquid droplet behavior on the plate surface at the threshold fluence of $0.6 \mathrm{~J} \mathrm{~cm}^{-2}$ is shown in Fig. 4. Most of the liquid droplet remained at the original position on the surface, even after 5-pulse laser irradiation (Figs. 4(b)4(d)). Some micro-vapor bubbles were produced in the liquid at the original position by the laser irradiation.

As the laser with a wavelength of $1053 \mathrm{~nm}$ was not photo-absorbed by the liquid (the absorption spectrum of the liquid is shown in Fig. 5), the laser beam was able to reach the surface of the aluminum plate through the transparent liquid, as shown in Fig. 1. A rapid temperature increase caused by the laser irradiation would occur at the surface of the aluminum plate. Boiling of the transparent liquid was induced at the interface between the metal plate and the droplet because the boiling point of the liquid (ethyl-methyl-carbonate (b.p. $=107^{\circ} \mathrm{C}$ ) and ethylenecarbonate (b.p. $\left.=244^{\circ} \mathrm{C}\right)$ ) was much lower than the melting point of aluminum (m.p.: $660^{\circ} \mathrm{C}$ ).

When the laser irradiation was performed with a fluence of $0.8 \mathrm{~J} \mathrm{~cm}^{-2}$, explosive boiling at the interface induced desorption of the droplet from the plate. The present results suggest that an optimized laser fluence below the surface damage threshold of the aluminum plate can effectively induce single-pass cleaning of large liquid droplets from a metal surface.

Figures 6 and 7 show shallow morphological damage observed in the droplet-ejected area of the metal surface after liquid ejection at a fluence of $0.8 \mathrm{~J} \mathrm{~cm}^{-2}$. These phenomena suggest that the incident laser beam may have been slightly focused by the droplet onto the metal surface. When the laser irradiation was performed at fluences above $1 \mathrm{~J} \mathrm{~cm}^{-2}$, both severe damage to the metal surface and liquid vaporization were observed.

\section{Summary}

We investigated laser-induced ejection of a millimetersized liquid droplet from an aluminum metal surface with a high-power ns-laser. The original droplet was successfully eliminated from its initial position on the surface with negligible damage to the laser-irradiated area of the metal. Using high-power laser equipment, we have demonstrated that it is possible to perform non-contact single-pass cleaning of a large area on a solid surface in ambient atmosphere.

\section{Acknowledgments}

We are grateful to Dr. N. Okada of the Corporate Manufacturing Engineering Center, Toshiba Corp. for helpful discussions. This work was funded by the Impact Program "Ubiquitous Power Laser for Achieving a Safe, Secure and Longevity Society (Program Manager: Dr. Yuji Sano)” of the Council for Science, Technology, and Innovation (Cabinet Office, Government of Japan).

\section{References}

[1] D. Bauerle: "Laser Processing and Chemistry (4th Ed.)”, (Springer-Verlag, Berlin \& Heidelberg, 2011) Chapter: 23.7, pp.549-559.

[2] A. C. Tam, W. P. Leung, W. Zapka, W. Ziemlich: J. Appl. Phys.,71, (1992) 3515.

[3] O.Yavas, P. Leiderer, H. K. Park, C. P. Grigoropoulos, C. C. Poon, W. P. Leung, N. Do, A. C. Tam: Phys. Rev. Lett., 70, (1993) 1830.

[4] Y. Dou, L. V. Zhigilei, N. Winograd, B. J. Garrison: J. Phys. Chem., 105, (2001) 2748.

[5] K. Tagawa and R. J. Brodd: "Production Processes for Fabrication of Lithium-Ion Batteries (The Chapter 8 of "Lithium-Ion Batteries"), eds. by M. Yoshio, R. J. Brodd, and A. Kozawa”, (Springer-Verlag, New York, 2009) p.189.

(Received: July 6, 2018, Accepted: October 7, 2018) 